\title{
Activity in the SPB star 53 Piscium*
}

\author{
J.-M. Le Contel, P. Mathias, E. Chapellier, and J.-C. Valtier \\ Observatoire de la Côte d'Azur, Département Fresnel, UMR 6528, BP 4229, 06304 Nice Cedex 4, France
}

Received 6 March 2001 / Accepted 4 October 2001

\begin{abstract}
New spectroscopic observations of the star 53 Psc are presented. A correlation technique has been used to derive the radial velocity curves with a high accuracy. Four frequencies have been detected in the radial velocity variations using four frequency analysis algorithms. Three frequencies $\left(1.81,1.22\right.$ and $\left.1.57 \mathrm{~cd}^{-1}\right)$ are typical SPB star frequencies, the last one being close to our detection threshold. They are associated to low amplitude velocities, of the order of $1-2 \mathrm{~km} \mathrm{~s}^{-1}$. So we classify 53 Psc in the SPBs class in agreement with its position in the HR diagram. Line equivalent width and central depth variations are also observed. Two phenomena appear: first, a smooth variation, present in all lines with a time scale of 10 days, could be related to the stellar rotation and a magnetic field. Second, on two consecutive nights, a sudden and important increase (20\%) of equivalent widths and central depths of $\mathrm{H} \delta$ and $\mathrm{He}$ I lines could be due to an outburst similar to the ones present in some Be stars. The variation in the equivalent widths is mainly due to the increase of the central depth of the quoted lines. This suggests the existence of NLTE effects due to a hot low density plasma in the upper layers of the atmosphere of $53 \mathrm{Psc}$. An additional frequency is pointed out around $0.11 \mathrm{~cd}^{-1}$. The best interpretation is an SPB frequency shifted by rotation.
\end{abstract}

Key words. stars: individual: 53 Piscium - stars: oscillations - stars: variables: general - stars: activity

\section{Introduction}

Several theoretical papers (see e.g. Pamyatnykh 1999 and references therein) show that the instability strips corresponding to $\beta$ Cephei and SPB stars have an intersection zone, allowing the possibility for a star present in this region to display characteristics of these two classes. Candidates should be looked for in the B 2-B 3 region of the HR diagram. Observationally, two different time scales should be simultaneously present: one corresponding to SPB frequencies, of the order of $1 \mathrm{~cd}^{-1}$, and one corresponding to $\beta$ Cephei frequencies, of the order of $5 \mathrm{~cd}^{-1}$. Monitoring such stars, with the presence of simultaneously excited $p$ - and $g$-modes, is of particular importance since both the outer and inner layers can be probed. A first attempt concerned the B 3 star $\iota$ Herculis (Chapellier et al. 2000), which has been classified as an SPB star with sometimes, transiently, the presence of high frequencies. However, no definitive values for these latter have been derived from the data. It was therefore useful to consider another candidate.

Send offprint requests to: P. Mathias,

e-mail: mathias@obs-nice.fr

* Based on observations obtained at the Observatoire de Haute-Provence.
The star 53 Piscium (HD 3379, $V=5.86, \mathrm{~B} 2.5 \mathrm{IV}$ ) is a good target from its location in the HR diagram. Its variability was first reported by Williams (1954) who derived a frequency of the order of $11 \mathrm{~cd}^{-1}$ from photometric observations. Later, Mathews (1956) could not derive any periodicity in radial velocities but pointed out that variations were indeed present. But Percy (1971) found the star to be constant in photometry. New photometric and spectroscopic data allowed Sareyan et al. (1979) to classify $53 \mathrm{Psc}$ as a $\beta$ Cephei star, with a frequency of $12.5 \mathrm{~cd}^{-1}$ in light variations, whereas the velocity variations led to a frequency around $18.2 \mathrm{~cd}^{-1}$, estimated from the position of two maxima. Then, from photometric observations obtained on a single night in 1985, Wolf (1987) derived a $10.4 \mathrm{~cd}^{-1}$ frequency corresponding to the largest amplitude ever recorded on that star (0.035 mag in the $V$ band). Following this publication, Le Contel et al. (1988) and Balona \& Marang (1988), both from long term photometric monitoring, could not find any significant periodic variation with an amplitude larger than $0.002 \mathrm{mag}$. Finally, Jerzykiewicz \& Sterken (1990) found that 53 Psc was constant and attributed the photometric variations to the usual comparison star, 34 Psc. They generalised this hypothesis to all previous photometric studies except that of Wolf (1987) which remains unique in this sense. 


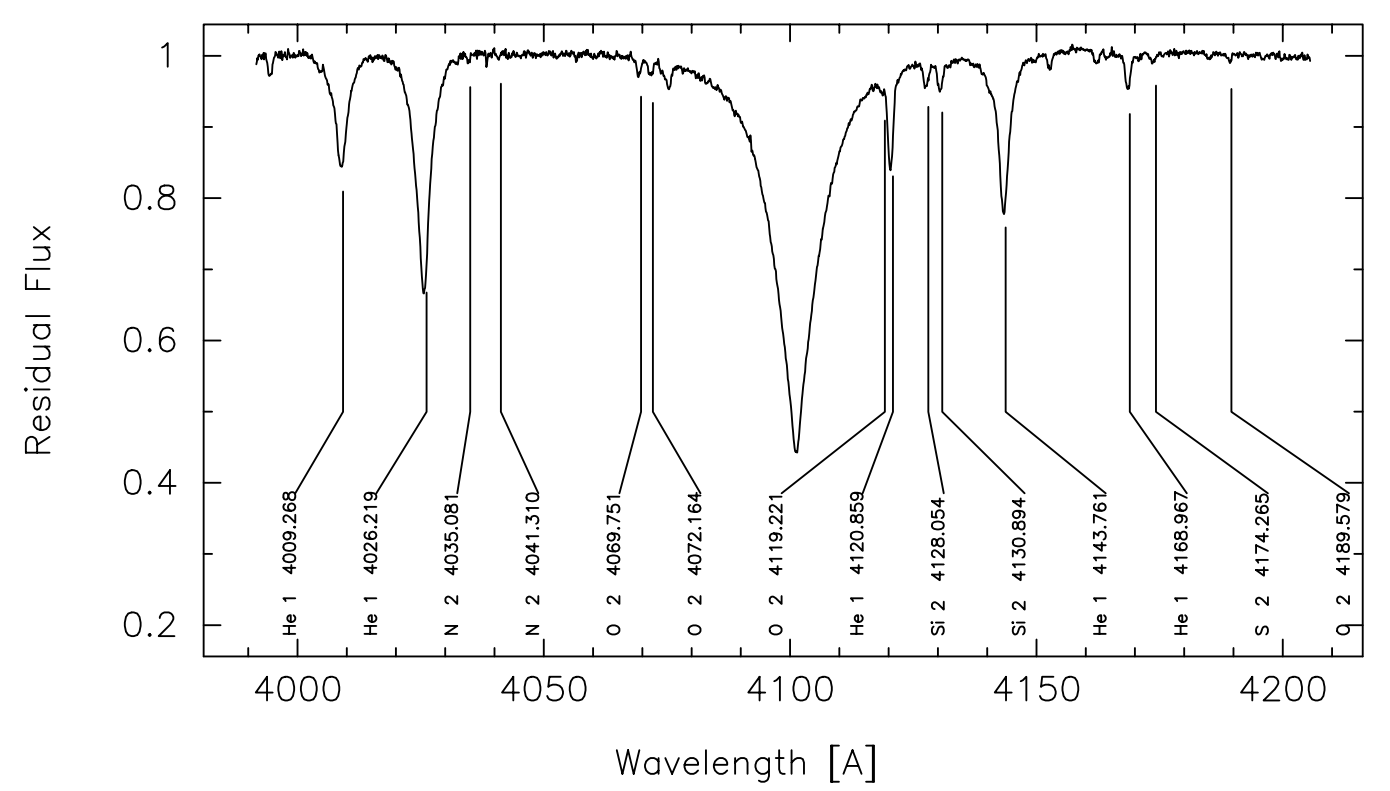

Fig. 1. Spectrum of 53 Psc obtained on September 2, 1996. Main lines have been identified.

The aim of the present paper is to improve our understanding of 53 Psc using new spectroscopic data (described in Sect.2). Radial velocities are discussed in Sect. 3. Frequency analyses of our and Hipparcos data are performed in Sect. 4. The nature of the variations of $53 \mathrm{Psc}$ is discussed in Sect. 5. Then, the particular behaviour of equivalent widths and central depths is presented and interpreted in Sect. 6. Finally, some conclusions are given in Sect. 7.

\section{Observations and data reductions}

The observations were obtained in 1996 with the spectrograph AURELIE (Gillet et al. 1994), at the Coudé focus of the $1.52 \mathrm{~m}$ telescope of the Observatoire de HauteProvence. The detector was a one-dimensional CCD. The observations spread over 11 nights, between August 30 and September 9. Due to bad weather conditions, nothing was measured during the night of September 3. The spectral domain covered $214 \AA$ and was centered around $\mathrm{H} \delta$. It contains about 20 identified lines of different elements, i.e. H $\delta$, He I, Si II, O II, S II, N II. . S See Fig. 1. The resolving power was around 15000 . To ensure a signal-to-noise ratio above 200, the average exposure time was around $20 \mathrm{~min}$. More than 100 stellar spectra were obtained this way.

Each spectrum has been corrected for the pixel-topixel response by flat-field and offset spectra. The wavelength calibration was based upon about 30 lines of a thorium lamp. Finally, the continua were fitted by a cubic spline function, and the spectra were normalized by this function.

\section{Radial velocity curves}

As important differences exist in the intensities and shapes of the different lines, radial velocities determined from the absolute positions of a small number of lines lead to a rather large dispersion ( $\sigma$ of the order of $2 \mathrm{~km} \mathrm{~s}^{-1}$ after correction for the observed systematic shifts in the He I lines velocities, see Ducatel et al. 1981). So, as only low amplitude variations were expected, and following Mathias \& Aerts (1996), we applied a correlation algorithm to determine more accurate radial velocities. The advantages of this technique are the following: it is a differential method allowing comparison between a reference spectrum and a given one and it takes into account the complete spectrum (here 2048 pixels) and all the detected lines in the domain.

We define an auto-correlation function $f(v)$ as

$f(v)=(1-g(v)) \times(1-r(v))$

where $g(v)$ is a spectrum, $r(v)$ the reference spectrum, and $v$ a trial velocity. In order to get a sharper $f$-function, spectral lines as well as continuum are considered i.e., the whole spectrum. This function is computed by varying $v$ from -40 to $+40 \mathrm{~km} \mathrm{~s}^{-1}$ with a $0.5 \mathrm{~km} \mathrm{~s}^{-1}$ step. Finally, the $f$-function is fitted with a Gaussian, the center of which provides the velocity attributed to the atmospheric motion. Such a procedure leads to velocities in the frame of the reference spectrum. This latter is selected as the one having the highest signal-to-noise ratio $(>300)$ together with the most symmetrical profiles. These conditions are fulfilled for a spectrum obtained on the night of September 2 and is represented in Fig. 1. Since the $f$-function provides velocities in the reference spectrum frame, we attributed to this latter the velocity concerning the Si II doublet. Finally, the velocity curves shown in Fig. 2 are obtained.

Note that a few irrelevant points were removed, in particular two points on the first night (the corresponding spectra have a different central wavelength, so the spectral domain is not the same), and the points corresponding to spectra having a bad signal-to-noise ratio (below 50). 


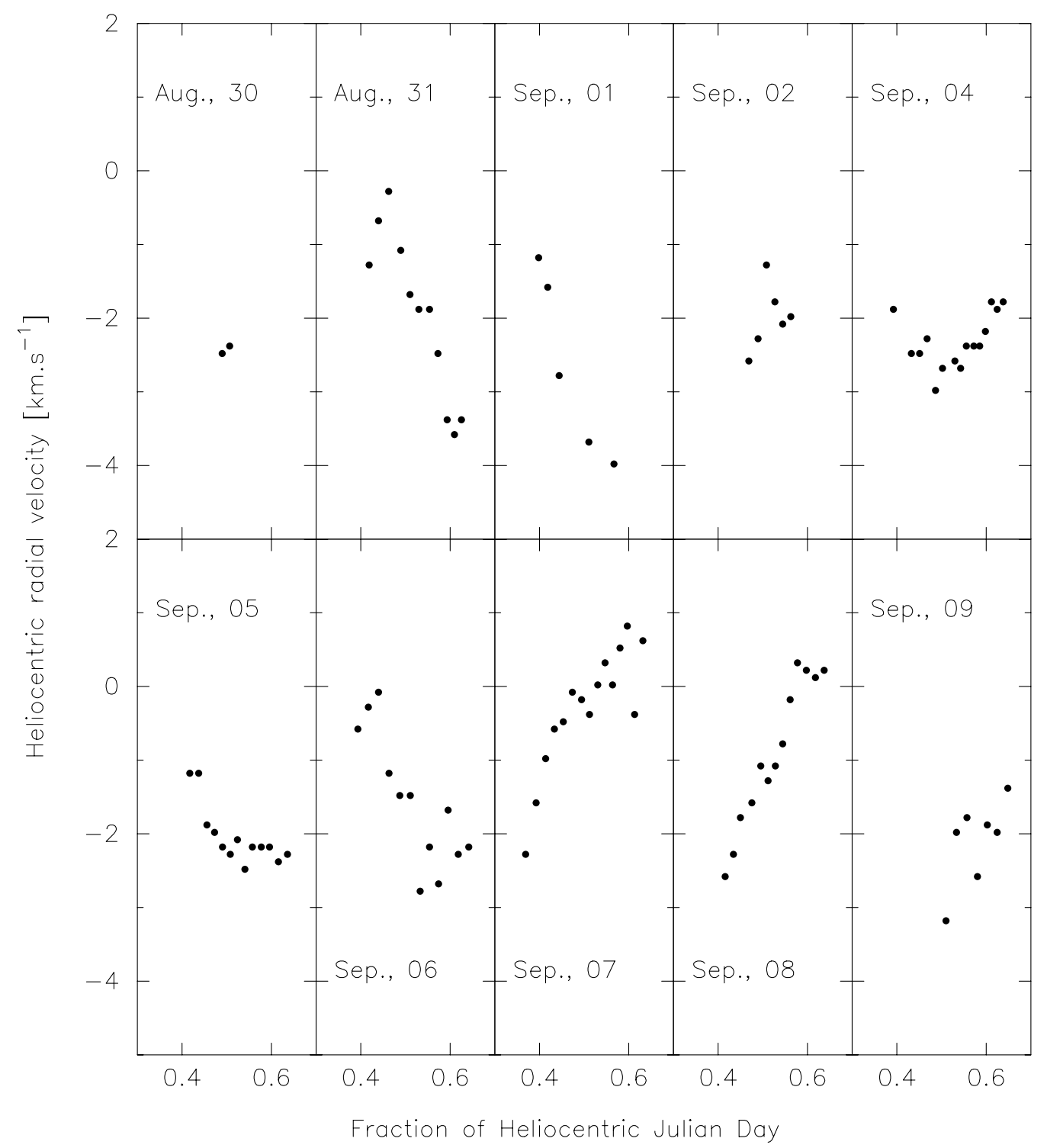

Fig. 2. Radial velocity obtained from the auto-correlation function.

Indeed, the method used here is very sensitive to this parameter since the complete spectrum is used: the uncertainty in the location of both lines and continuum affects the shape of the $f$-function, hence also the deduced velocity.

The scatter is estimated to be about $\pm 0.4 \mathrm{~km} \mathrm{~s}^{-1}$. The velocity range within a night is about $3 \mathrm{~km} \mathrm{~s}^{-1}$, but can also be less than $1 \mathrm{kms}^{-1}$ (September 5$)$. The mean velocity is around $-1.6 \mathrm{~km} \mathrm{~s}^{-1}$.

\section{Frequency analysis}

The only three spectroscopic works concerning this star are those of Mathews (1956), Abt et al. (1990) and Sareyan et al. (1979). Except for Abt et al. (1990), these studies reveal velocity variations, but none was able to directly derive a definite periodicity. Our velocity curves present clear variations on every night.
Four different methods, adapted to unequally spaced data, were used to perform the frequency analysis: Fourier, CLEAN (Roberts et al. 1987), Vanicek (1971) and PDM algorithms (Stellingwerf 1978). A recurrent problem is the choice between the real frequency and its aliases. We selected the frequency using 4 ways that led to coherent results: the two first consist of a graphical inspection of the phased data and an inspection of the fit within these data, the two other favour the one having the largest amplitude and the smallest residuals from a sine-fit.

Power spectra are presented in Fig. 3. On each frequency distribution, a main peak arises around $1.81 \mathrm{~cd}^{-1}$. This $\nu_{1}$ frequency accounts for $45 \%$ of the variance computed from a sine-fit. The $2 \mathrm{~K}$ associated amplitude is $2.0 \mathrm{~km} \mathrm{~s}^{-1}$.

However, a relatively large dispersion remains, and $55 \%$ of the supposed sine variation are not taken into account. We performed an additional frequency analysis on the data set prewhitened with the $\nu_{1}$ frequency. 


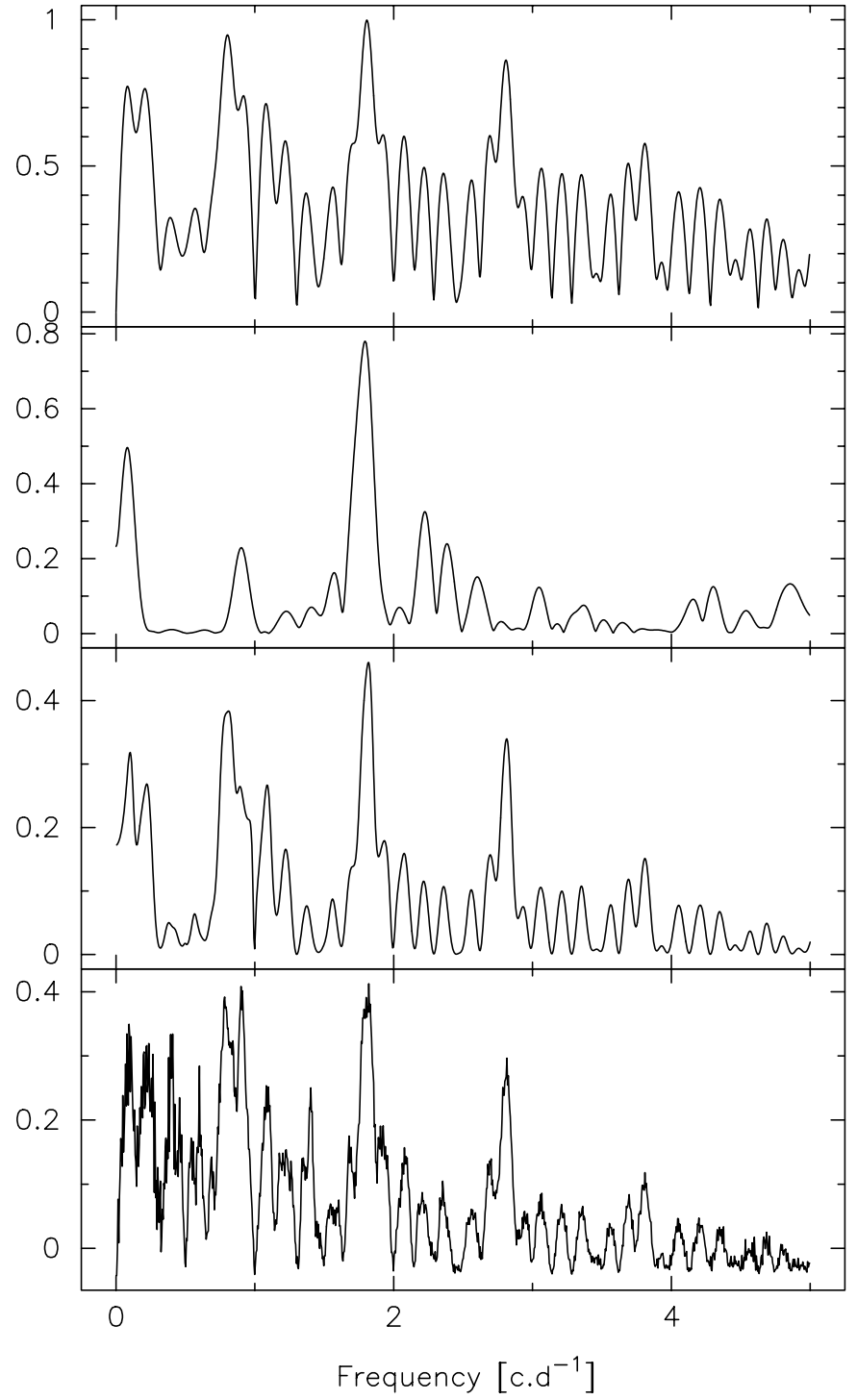

Fig. 3. Frequency analyses performed on the correlation velocity data set represented in Fig. 2. From top to bottom: Fourier periodogram; power spectrum obtained after 100 iterations with the CLEAN algorithm (gain is 0.5); power spectrum obtained from Vanicek's method; periodogram obtained from the PDM method with a $(5,2)$ bin structure (for convenience $(1-\theta)$ is represented).

The derived power spectra are represented in Fig. 4. The dominant peak is $\nu_{2}=0.11 \mathrm{~cd}^{-1}$ and is present whatever the method used. But, since the corresponding period $(9 \mathrm{~d})$ is of the order of the length of our observations (11 d), the $\nu_{2}$ value is doubtful. However, it is clear from Fig. 2 that a remaining low frequency is present. Too small to be attributed to a typical B-star pulsation, $\nu_{2}$ will be discussed in Sect. 6.

A new prewhitening, using $\nu_{1}$ and $\nu_{2}$, leads to the periodograms presented in Fig. 5 . The main peak provides the $\nu_{3}=1.22 \mathrm{~cd}^{-1}$ frequency, which accounts for $36 \%$ of the variance in the residuals, corresponding to a $2 K$ associated amplitude of $1.1 \mathrm{~km} \mathrm{~s}^{-1}$.

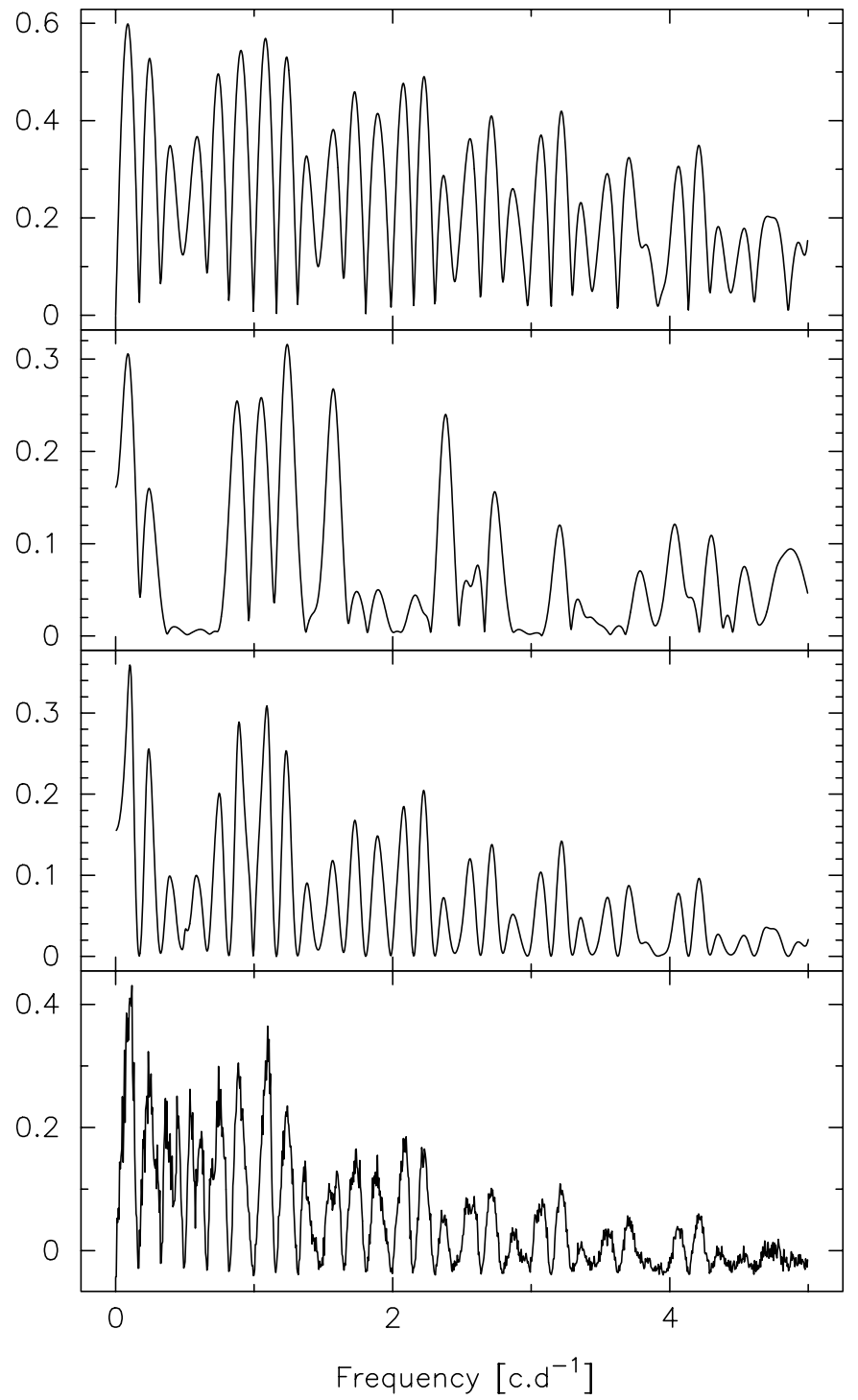

Fig. 4. Same as Fig. 3, after prehwitening the data set with $\nu_{1}=1.81 \mathrm{c} \mathrm{d}^{-1}$.

Table 1. Results provided by the sine-fit with the 4 detected frequencies simultaneously applied to the correlation data set. Columns represent respectively the frequency, the $2 \mathrm{~K}$ amplitude and the associated fraction of the variance. The residuals (rms) are $0.37 \mathrm{~km} \mathrm{~s}^{-1}$, the uncertainty on the $2 K$ amplitudes is $0.1 \mathrm{~km} \mathrm{~s}^{-1}$.

\begin{tabular}{ccr}
\hline Frequency $\left[\mathrm{cd}^{-1}\right]$ & $2 K\left[\mathrm{~km} \mathrm{~s}^{-1}\right]$ & Var $[\%]$ \\
\hline$\nu_{1}=1.81$ & 2.24 & 36 \\
$\nu_{2}=0.11$ & 1.92 & 23 \\
$\nu_{3}=1.22$ & 1.38 & 16 \\
$\nu_{4}=1.57$ & 0.92 & 9 \\
\hline
\end{tabular}

After prewhitening the residuals with $\nu_{3}$, we obtained the power spectra represented in Fig. 6 .

A main peak still appears, whatever the method used, at $\nu_{4}=1.57 \mathrm{~cd}^{-1}$. This latter frequency again accounts for $32 \%$ of the variance in the residuals, and the 


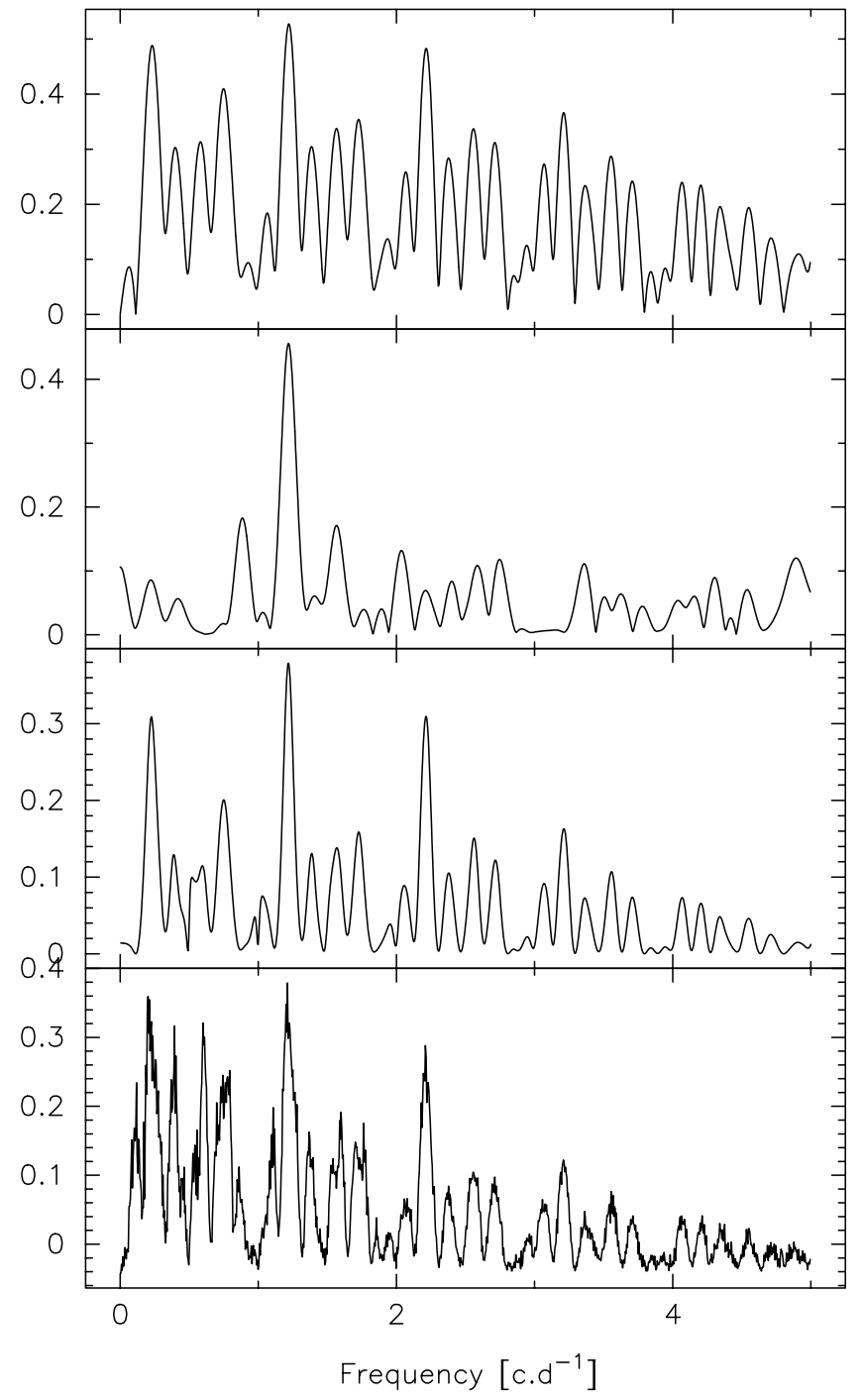

Fig. 5. Same as Fig. 3, after prehwitening the data set with both $\nu_{1}$ and $\nu_{2}$.

associated $2 K$ amplitude is $0.8 \mathrm{~km} \mathrm{~s}^{-1}$. An additional prewhitening leads to peaks lower than the detection threshold.

We applied the false-alarm test defined by Scargle (1982) on our frequency analysis. The $2 K$-amplitude limit value is $1.36 \mathrm{~km} \mathrm{~s}^{-1}$ for a $1 \%$ confidence level, considering a 1- $\sigma$ dispersion of $0.2 \mathrm{~km} \mathrm{~s}^{-1}$ on the data and about 1000 trial frequencies. Therefore, frequencies corresponding to amplitudes above this limit have a $99 \%$ probability to be attributed to a signal and not to noise. From Table 1, the 3 first frequencies are above this limit and can thus be considered as real, while the last one is below this confidence level. In order to visualize the reality of the 4 detected frequencies, radial velocity data, prewhitened with 3 frequencies, have been represented as a function of phase computed from the remaining frequency. The result is represented in Fig. 7. The first 3 frequencies are well seen on the curves, but as expected the last one is poorly visualized.

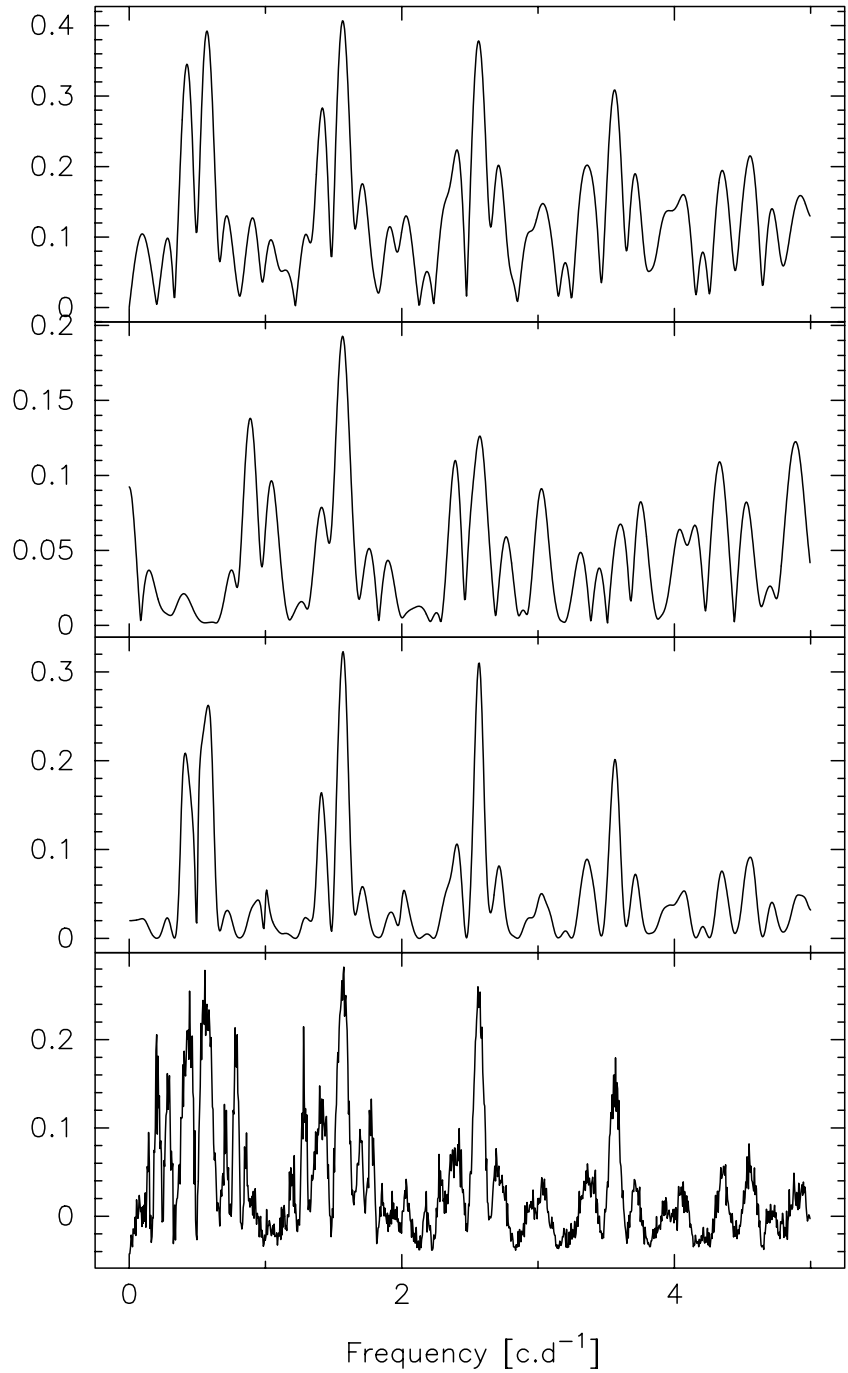

Fig. 6. Same as Fig. 3, after prehwitening the data set with the $\nu_{1}, \nu_{2}$ and $\nu_{3}$ frequencies.

A sine-fit computed with the 4 detected frequencies simultaneously shows that they account for more than $80 \%$ of the total variation (see Table 1 ). Other frequencies could be present. The radial velocity data together with the sine-fit using these 4 frequencies are represented in Fig. 8.

Following the procedure used in the case of $\iota \mathrm{Her}$ (Chapellier et al. 2000), the 53 Psc Hipparcos data (ESA 1997), which consist in 97 observations recorded through both $B_{\mathrm{T}}$ and $V_{\mathrm{T}}$ filters, were analyzed. The maximum peak in the CLEANed periodogram (0.5 gain, 100 iterations) has a peak around 0.005 mag i.e., under the detection threshold. Consequently, as already pointed out by Percy \& Au-Yong (2000), no frequency can be derived from Hipparcos data.

\section{Discussion}

\subsection{Classification of 53 PsC}

The spectral type of $53 \mathrm{Psc}$ is given to be $\mathrm{B} 2.5 \mathrm{IV}$ (Lesh 1968) from which a mass of $6 M_{\odot}$ can be derived (Andersen 1991) and consequently 


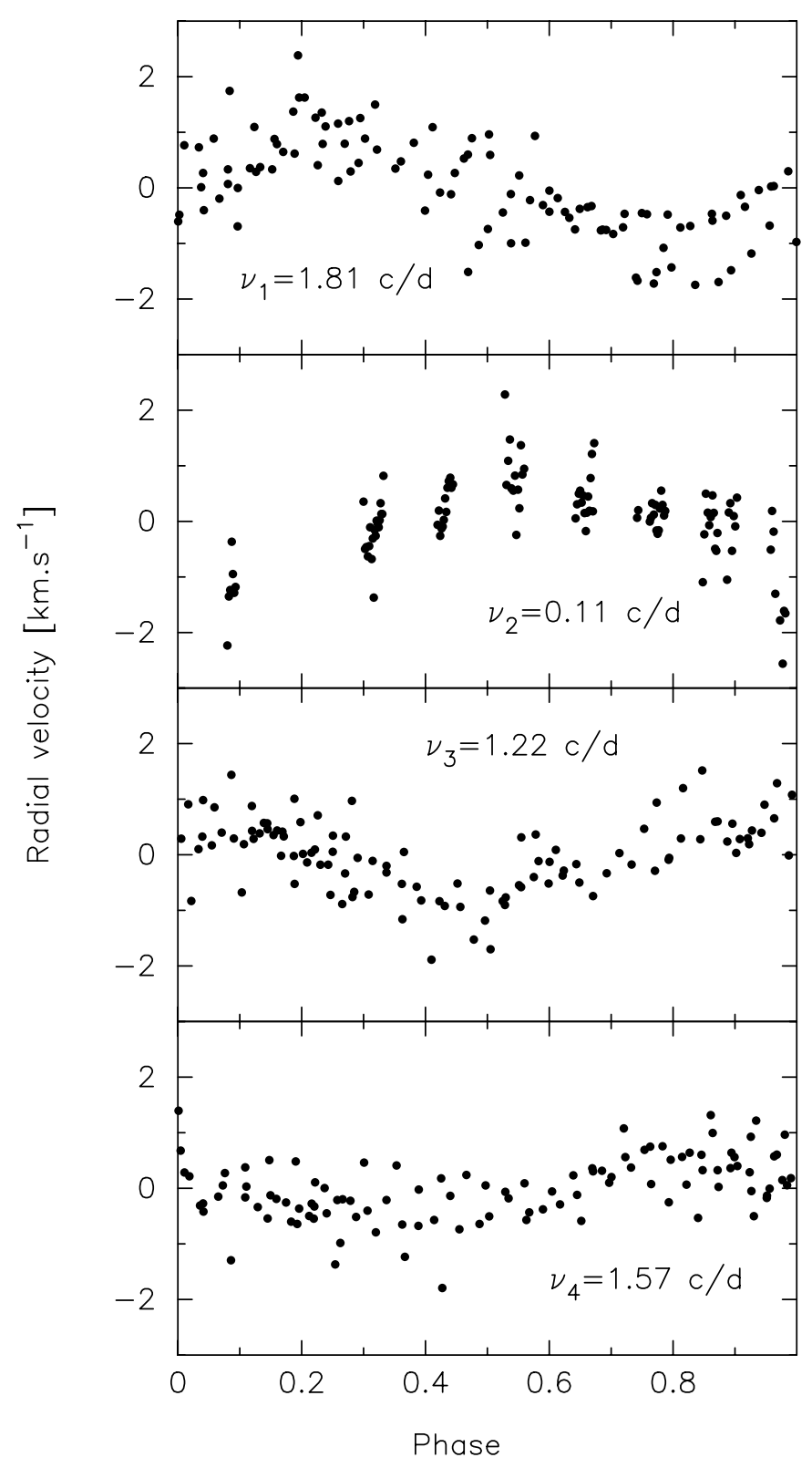

Fig. 7. The radial velocities, prewhitened with three out of four frequencies, are represented as a function of phase for the remaining one as indicated on the graph.

$\left(\log L / L_{\odot}, \log T_{\text {eff }}\right)=(3.17,4.25)$. Note that from the photometric calibrations by Künzli et al. (1997), we get $\left(\log L / L_{\odot}, \log T_{\text {eff }}\right)=(2.92,4.24)$. Thus, 53 Psc lies in the SPB instability strip (e.g. Gautschy \& Saio 1996). Since it is also multiperiodic with frequencies typical for SPBs, 53 Psc should be classified as an SPB star.

\subsection{Transient short period variations?}

As quoted in the introduction, several previous studies reported a very short time-scale in the variations of $53 \mathrm{Psc}$ : Williams (1954) gives $10.9 \mathrm{~cd}^{-1}$ in photometry, Sareyan et al. (1979) detected a $12.5 \mathrm{~cd}^{-1}$ in the light variations. From the non detection of such a large frequency in other studies together with the analysis of new data,

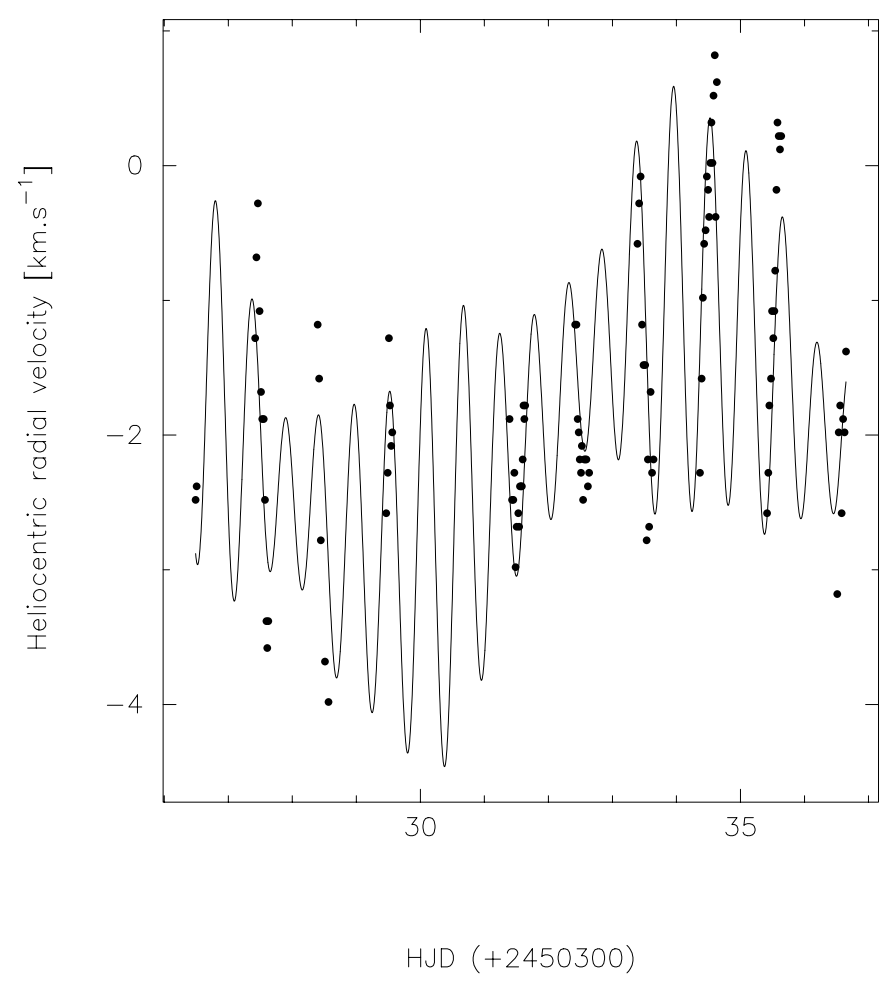

Fig. 8. Superposition of the radial velocity data (dots) with the sine-fit computed using the 4 frequencies provided in Table 1.

Jerzykiewicz \& Sterken (1990) attributed this frequency to one comparison star, 34 Psc (B9 V). It follows that the only clear detected rapid variations that can be attributed to 53 Psc itself are from the photometric work of Wolf (1987) who recorded a large amplitude associated to this large frequency. From a spectroscopic point-of-view, rapid variations have only been detected in Sareyan et al. (1979).

Our data set did not show any rapid variations. After prewhitening with the 4 detected frequencies, a frequency analysis has been performed up to a $30 \mathrm{~cd}^{-1}$ value. No peak above the detection thereshold appears. In addition, power spectra computed for each individual night do not present any significant peak.

The spectral classification of 53 Psc given in Sect. 5.1 makes this star close to $\iota$ Herculis (Chapellier et al. 2000) in the HR diagram. Consequently, 53 Psc may similarly show the same invoked transient variability, a possibility also suggested by Balona \& Marang (1988). Such a behaviour has been suspected in 22 Ori, a slightly hotter variable B star (Balona \& Engelbrecht 1985).

\section{Long-term variation}

\subsection{Equivalent width and central depth variations}

Equivalent widths and central intensities of SiII, HeI and $\mathrm{H} \delta$ lines have been measured. They vary from night to night as shown in the case of the $\mathrm{H} \delta$ line presented in Fig. 9. Note that because other lines are in the large wings of $\mathrm{H} \delta$, the integration was computed between $4088 \AA$ and $4114 \AA$. Thus the presented equivalent width 


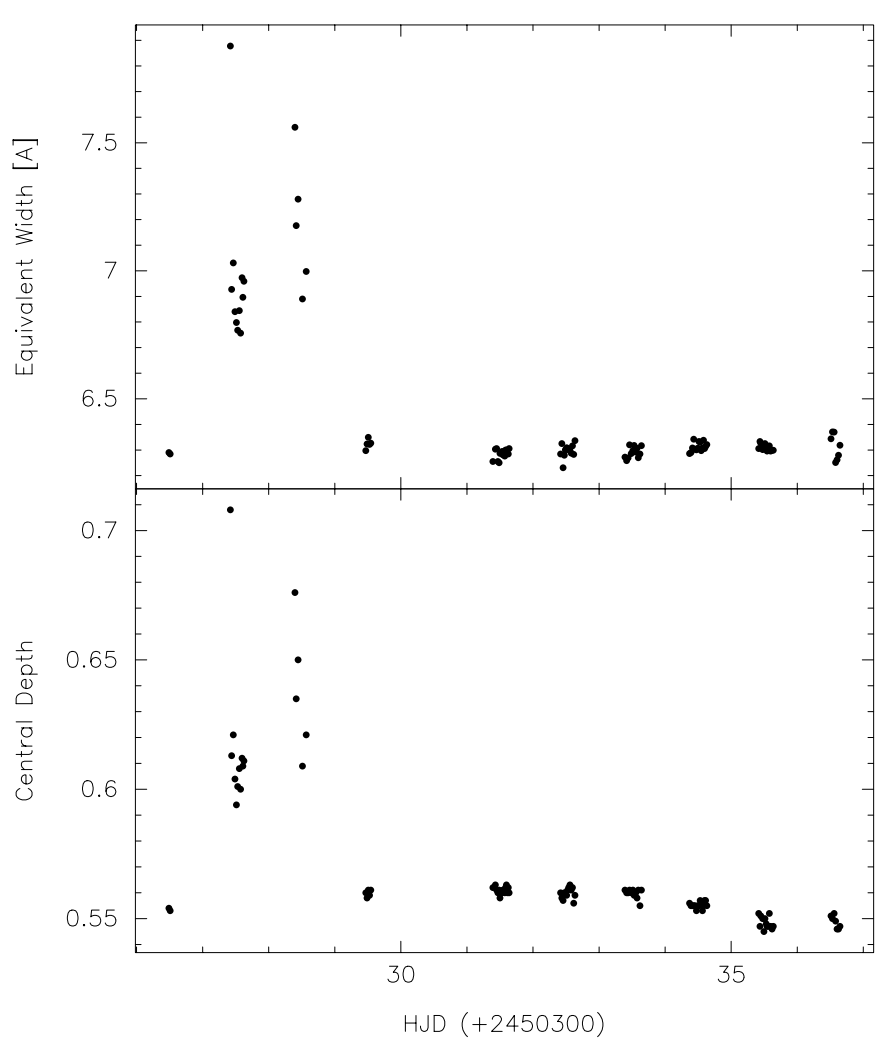

Fig. 9. Upper panel: equivalent width of the $\mathrm{H} \delta$ line as a function of time. Lower panel: central depth as a function of time for the $\mathrm{H} \delta$ line.

is a lower limit only. We are confident that these variations cannot be attributed to normalisation errors, as can be seen in Fig. 10. In precedent studies, Mathews (1956) could not see any correlation between line profile and velocity variations, whereas Sareyan et al. (1979) observed that the behaviour of the lines was similar to those recorded in different $\beta$ Cephei stars. It is obviously not the case here.

Two phenomena are present:

i) A smooth variation of a few percent is particularly visible in the central depth variations, a change also present in the HeI lines and in the faint Si II doublet. This variation has a clear maximum around HJD 2450331, whereas a possible minimum is present around HJD 2450336. That suggests a time scale of the order of 10 days. Usually, non-radial pulsations hardly affect the equivalent width of a line profile, particularly in the case of the SPB stars where amplitudes are very low. Conversely, long-period changes in the line-profile behaviour may be due to rotation when surface inhomogeneities occur. Sareyan et al. (1979) determined $v \sin i=30 \mathrm{kms}^{-1}$ and Andersen (1991) a $4 R_{\odot}$ for a B2.5 V star. These two data provide an upper limit of the rotational period of the order of $7 \mathrm{~d}$. However, considering the character slightly more evolved of $53 \mathrm{Psc}$, and the errors affecting each term, this value is still compatible with the time scale of $10 \mathrm{~d}$ for the rotation discussed here. These surface anomalies require a magnetic field;

ii) A strong (about 20\%) and sudden increase of the same parameters appears on the second and third nights in $\mathrm{H} \delta$ and He I lines. This behaviour is apparently not seen on the Si II doublet lines nor on any faint line. Although these lines actually present an increase in their equivalent widths, it is less than $5 \%$, well below the $20 \%$ increase observed for $\mathrm{H} \delta$ and helium lines. Such a sudden (and possibly not periodic) event has never been reported before in spectroscopy nor in photometry for $53 \mathrm{Psc}$. This phenomenon cannot be accounted for by a temperature increase of the star as the sensitivity to temperature variations of hydrogen and helium lines are anticorrelated for a B 3 star. An attractive hypothesis could be flares, as observed in some Be stars (see e.g. Smith \& Robinson 1999). However, flares are usually located on a relatively small area on the stellar surface. Hence, the ejected matter should have a privileged direction, thus a definite Doppler velocity, implying asymmetric profiles. However, profiles during the abnormally large equivalent width phase remain symmetric for $\mathrm{H} \delta$ at least (Fig. 10), whereas the signal-to-noise ratio for other lines are not high enough to conclude to perfect symmetries for the profiles. Consequently, the mechanism should concern the whole visible hemisphere of the star.

According to Underhill \& Doazan (1982), such a strengthening of the cores of the He I lines arising from the $2^{3} P_{0}$ level (such as $\lambda \lambda 4026$ and 4121) is a diagnostic criterion for the presence of a hot, low-density plasma in the outer parts of the star. Presently the observed effects on the line profiles (increase of the central intensities) could arise from NLTE effects. The fact that Si II lines are not (or very marginally) affected can be explained by their lower sensitivity to non-LTE effects. Consequently, this phenomenon could be related to an outburst similar to the ones observed in Be stars. These short lived outbursts are more frequently observed in early Be stars (B0 to B 4) with rather low $v \sin i$ (Hubert \& Floquet 1998). As nothing appears in the 53 Psc Hipparcos photometric data, the increase of equivalent widths observed in our data is either exceptional (such as the large photometric amplitude detected on one night by Wolf 1987) or indicates the beginning of an important activity.

Nevertheless, if both rotational modulation and activity interpretations seem to require a magnetic field, the presence of this latter has to be proved for $53 \mathrm{Psc}$.

\subsection{Radial velocity variations}

As shown in Sect. 4, a long term variation is present, with a frequency $\nu_{2}=0.11 \mathrm{~cd}^{-1}$. Despite its actual value could be somewhat different due to the too short time coverage of our data, the associated variation is clearly above the false-alarm probability of $1 \%$. Such a frequency may 
originate from 3 possible mechanisms: binarity, rotation and pulsation that we examine hereafter.

The first interpretation is that this long period is due to an orbital motion. The main objection to this hypothesis is the low amplitude $\left(2 K=1.92 \mathrm{~km} \mathrm{~s}^{-1}\right)$ for a 9 -d period. The corresponding mass function is about $0.04 M_{\odot} / \sin ^{3} i$. From the above-mentioned interpretation of the central depth and equivalent width variations, the star should be seen rather equator-on than pole-on. Except if all modes are axi-symmetric, this geometry also favours the detection of non-radial modes. But even if the inclination angle is $45^{\circ}$, the mass of the companion is still very low, being about $0.05 M_{\odot}$. Abt et al. (1990) ruled out the binarity hypothesis for $53 \mathrm{Psc}$, but their data are much more sparse than ours.

The second interpretation deals with rotational modulation: indeed, if inhomogeneities are present on the stellar surface, they should affect line profile variations, not only with regards to the equivalent width or the central depth, but also with the profile symmetry. However, our profiles have signal-to-noise ratios which are not high enough to allow the monitoring of the displacement of a bump during the rotational period. Nevertheless, in view of Sect. 6.1, this hypothesis should not be rejected.

The last interpretation refers to pulsation. The $\nu_{2}$ frequency is clearly out of the frequency range $0.25-2 \mathrm{~cd}^{-1}$ computed by Pamyatnykh (1999) for SPBs. But these "theoretical" frequencies are provided in the stellar rest frame. The derivation of these frequencies from the observed ones requires a correction due to the stellar rotation. For high order $g$-modes, which are the ones excited in SPBs, an observed frequency $\nu_{\text {obs }}$ will be related to the co-moving frequency $\nu_{0}$ by (Cox 1980):

$\nu_{\mathrm{obs}}=\nu_{0}-m\left(1-\frac{1}{\ell(\ell+1)}\right) \Omega$

where $\Omega$ is the stellar rotation frequency. The frequency shift for an $(\ell, m)=(3,3)$ mode amounts to $0.275 \mathrm{~cd}^{-1}$ if we retain the $10 \mathrm{~d}$ rotation period discussed in Sect. 6.1. In this hypothesis, the $\nu_{2}$ frequency becomes, in the comoving frame, $0.385 \mathrm{~cd}^{-1}$ i.e. a value within the normal range for an SPB star. If the rotation period is smaller, an $(\ell, m)=(2,2)$ mode plays the same role.

Among the three hypotheses discussed here, the last one seems the most credible, but the two others cannot be definitively excluded.

\section{Conclusion}

Our new observations show that the star 53 Psc is a multiperiodic variable with frequencies typical of SPBs variations. Consequently, and because it belongs to the corresponding instability strip, $53 \mathrm{Psc}$ should be classified as an SPB star.

We could not confirm any of the short-term variations recorded in some previous studies. The idea of transient variability, as mentioned sometimes, would be of the same nature as the one already invoked in the case of $\iota$ Her.

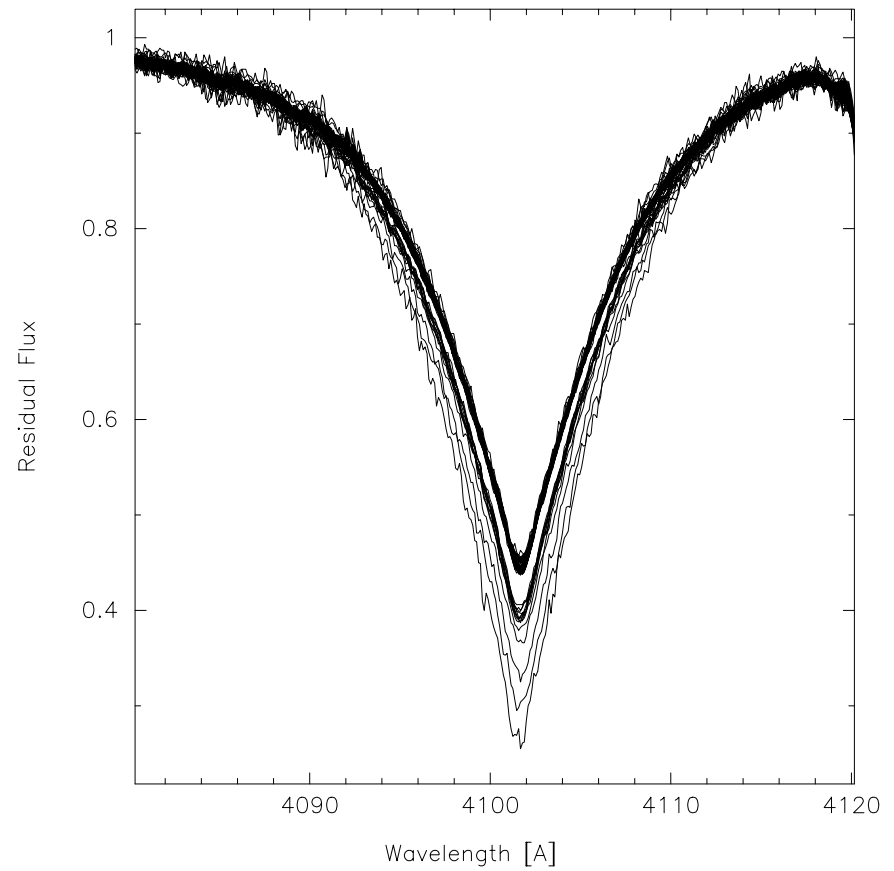

Fig. 10. Plot of all spectra around the $\mathrm{H} \delta$ line.

An additional frequency with a much lower value, $\nu_{2}$, is also detected. Three hypotheses are evoked: binarity, rotation modulation, and a displacement of the SPB frequency in the co-moving frame. A pulsation mode with $(\ell=3)$, shifted by rotation, seems the best interpretation, despite the fact that binarity and rotational modulation cannot be definitively ruled out. However, we adress the problem to numericians since in general only low-degree $(\ell=1$ or 2$)$ modes are studied for stability. It would be interesting to use these codes to at least $\ell=4$. Finally, note that this frequency displacement may also affect the other detected frequencies, but even for an $(\ell, m)=(3,3)$ mode, none can be shifted to a value outside the typical range of the ones encountered in SPB stars.

Rotational modulation could explain the smooth variations of the profiles, affecting all lines and well seen on the residual fluxes. In addition, activity is invoked to explain sudden large changes in equivalent widths of $\mathrm{H} \delta$ and helium lines. Because possible line asymmetries are below our detection threshold, we attributed this phenomenon to the existence of a hot, low density plasma in the outer layers of the star.

Clearly, additional observations are needed to confirm these hypotheses. Spectro-polarimetric observations will be performed in order to detect the magnetic field. The observed outburst being quite similar (although on a different time scale) to what is observed in some Be stars, it would be interesting to observe $53 \mathrm{Psc}$ at $\mathrm{H} \alpha$ and UV wavelengths in order to check whether the star is undertaking a cycle of transitions from a "normal" B star to a Be or shell phase.

Note: the correlation velocity data are available upon request. 
Acknowledgements. We thank Dr. G. Guerrero for the permission to use his code based on Vanicek's (1971) algorithm. We are grateful to Dr. Prof. C. Aerts and Dr. R. Garrido who helped us to improve this paper as well as to the anonymous referee. We are also grateful to Dr. C. Sterken for his critical reading.

\section{References}

Abt, H. A., Gomez, A. E., \& Levy, S. G. 1990, ApJS, 74, 551 Andersen, J. 1991, Astron. Astrophys. Rev., 3, 91

Balona, L. A., \& Engelbrecht, C. A. 1985, MNRAS, 214, 559

Balona, L. A., \& Marang, F. 1988, IBVS, No. 3157

Chapellier, E., Mathias, P., Le Contel, J.-M., et al. 2000, A\&A, 362,189

Cox, J. P. 1980, in Theory of Stellar Pulsation (Princeton University Press)

Ducatel, D., Le Contel, J.-M., Sareyan, J.-P., \& Valtier, J.-C. 1981, A\&AS, 43, 359

ESA 1997, The Hipparcos and Tycho Catalogues (ESA SP1200), Noordwijk: ESA

Gautschy, A., \& Saio, H. 1996, ARA\&A, 34, 551

Gillet, D., Burnage, R., Kohler, D., et al. 1994, A\&A, 108, 181
Hubert, A.-M., \& Floquet, M. 1998, A\&A, 335, 565

Jerzykiewicz, M., \& Sterken, C. 1990, A\&A, 227, 77

Künzli, M., North, P., Kurucz, R. L., \& Nicolet, B. A\&AS, 122,51

Le Contel, J.-M., Chapellier, E., Valtier, J.-C., et al. 1988, IBVS, No. 3131

Lesh, J. R. 1968, ApJS, 17, 371

Mathews, R. T. 1956, PASP, 68, 455

Mathias, P., \& Aerts, C. 1996, A\&A, 312, 905

Pamyatnykh, A. A. 1999, Acta Astron., 49, 119

Percy, J. R. 1971, AJ, 76, 1105

Percy, J. R., \& Au-Yong, K. 2000, IBVS, No. 4825

Roberts, D. H., Lehár, J., \& Dreher, J. W. 1987, AJ, 93, 968

Sareyan, J.-P., Le Contel, J.-M., Ducatel, D., \& Valtier, J.-C. 1979, A\&A, 72, 313

Scargle, J. D. 1982, ApJ, 263, 835

Smith, M. A., \& Robinson, R. D. 1999, ApJ, 517, 866

Stellingwerf, R. F. 1978, ApJ, 224, 953

Underhill, A. B., \& Doazan, V. 1982, B stars with and without emission lines, CNRS/NASA SP456, 64

Vanicek, P. 1971, Ap\&S, 12, 10

Williams, A. D. 1954, PASP, 66, 25

Wolf, M. 1987, IBVS, No. 3003 